\title{
Ontology based Interoperability Approach for Heterogeneous Mobile Cellular Networks
}

\author{
Hasni Neji \\ Innov'COM Lab, Higher School \\ of Communications of Tunis, \\ Sup'Com University of Carthage, \\ Tunis, Tunisia.
}

\author{
Ridha Bouallegue \\ Innov'COM Lab, Higher School \\ of Communications of Tunis, \\ Sup'Com University of Carthage, \\ Tunis, Tunisia
}

\begin{abstract}
Interoperability issues between heterogeneous networks generations begin by establishing a holistic understanding of cellular communication systems (a starting point for interoperability improvements).

To fulfill what already mentioned, we propose an ontological approach that expresses the cellular communication systems' concepts, classes, and properties in a formal and unambiguous way [1].

This approach is first to analyze the structure, inputs, and outputs of three different cellular technologies [2] and [3], performing a domain analysis (of this subset of technologies) and producing a feature model of the domain. Finally, building an Ontology capable of providing a common view of the domain, and providing an effective representation of relations between concepts in different cellular technologies. The final goal is to achieve interoperability between them.
\end{abstract}

\section{General Terms}

Ontology, feature modeling, Interoperability.

\section{Keywords}

Interoperability; domain analysis; feature model; UML; GSM; UMTS; LTE-A.

\section{INTRODUCTION}

Telecommunication research is typified by developing or improving individual aspects of mobile cellular communication networks. Examples include research into seamless roaming, frequency allocation, cellular network's security, frequency reuse, terrestrial and satellite cellular networks interoperability, handover, cellular network planning, network architecture design, etc... These individual aspects of mobile cellular communication necessitate that the telecommunication researcher understands the domain and provide any needed interface between different cellular networks, allowing them to understand each other and interoperate. The already mentioned condition may be realized via the use of ontology. Figure 1 illustrates some individual aspects of three mobile networks technologies (Global System for Mobile -GSM-, Universal Mobile Telecommunication Systems -UMTS-, and Long-Term Evolution Advanced-LTE-A).

The technologies are not chosen arbitrary, but intentionally representing a succession of generations belonging to the same family. The main purpose is to make changes, commonalities, and variabilities noticeable and to facilitate the distinction between them. In this case, it will be easy to solve the interoperability challenges within this subset of cellular networks' technologies. Moreover, the cellular networks ontology is a pilot one. It should be generic and nonproprietary. It will allow any cellular network technology to be incorporated. The framework will neither be established solely for use with a specific group of technologies, nor be implemented simply to support research work. However, it must account for technologies used to improve it.

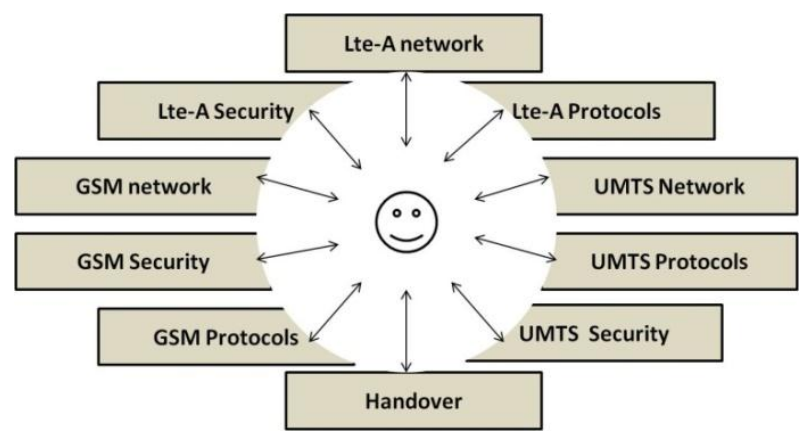

Figure 1: Individual aspects of mobile cellular communication networks.

\section{MOTIVATION}

The lack of interoperability between mobile cellular access networks has long been a challenging obstacle, which telecommunication researcher is trying to overcome. For instance, in second generation networks, this problem lies in the fact that there are multiple standards. Each of these standards can operate in the same frequency range. However, each utilizes a different Radio Technology and Modulation Scheme, which are characteristics of the standard. Therefore, the lack of interoperability in $2 \mathrm{G}$ occurs because of the lack of standardization.

Interoperability within $3 \mathrm{G}$ networks is limited to a few operating modes using different Radio Transmission Technologies that are not inter-operable. Thus, interoperability remains an issue for 3G. 4G technology even being successful in its various trials cannot guarantee the interoperability. This is within each network generation; meanwhile between heterogeneous networks' generations the situation seems to be worst. The reason that motivates us to develop an environment that maximizes interoperability, communication and efficiency tailored for this particular domain.

\section{APPLICATION OF ONTOLOGY FOR INTEROPERABILITY}

The main goal of this set of mind is to build an Ontology. The advantage of developing such specific ontologies tailored to the telecommunication's domain provides benefits stemming from representational efficiency. However, there has not been a lot of work in developing ontologies tailored to the domain of wireless cellular communication itself. One reason for this is the amount of effort required to produce such Ontology is 
substantial. Specific Ontologies such as this ongoing one are, in fact, not easily buildable. It will require from us to undertake seemingly heavy processes to identify existing features [4] in three different wireless cellular networks' technologies to satisfy the representational needs. An ideal solution will be offered by the construction of a general ontology for common features management, which might allow for resource sharing over and across multiple technologies in the telecommunication domain, possibly with an easy and fast process of customization without having to develop new systems from scratch.

The ontology should be extensible. Not only should it be possible to include new cellular technologies by using it, but it should also be possible to modify or update the attributes of the framework based on new technologies.

\subsection{Ontology overview}

The term "Ontology" is borrowed from philosophy where it is defined as a systematic investigation of "Existence". The term is now widely used in Artificial Intelligence and Knowledge Engineering where what "exist" is those entities which can be "represented." Ontology is the term used to refer to the shared understanding of some domain of interest that may be used as a unifying framework to solve problems in that domain [5]. Ontology necessarily entails or embodies a world view with respect to a given domain. This world view is often conceived as a set of concepts (e.g. entities, attributes, processes) along with their definitions and their inter-relationships.

Because people, organizations, and cellular technologies must communicate between and among themselves, there are often difficulties/inaccuracies in communications because of differing contexts, understandings, viewpoints and assumptions. Therefore, Ontologies help to accomplish the following:

- Improve poor communication,

- Establish a unifying framework for conceptual models and ideas,

- Establish the basis for interoperability, and

- Prevent redundant work and cross purposes.

\subsection{A proposed development strategy for ontology}

Our strategy for developing the Ontology will be based on both a top-down and bottom-up approach [6]. In order to be effective, we sought to make the top-down approach tackle the core problem of the interoperability between the cellular networks' technologies. The bottom-up approach will focus on developing specific cellular networks technologies' ontologies that accurately described the artifacts produced by the cellular networks' technologies, so that their data processes could be actually made to interoperate.

Cellular networks technologies' ontology is a system of features, selected because of their usefulness to capture interesting commonalities and similarities between technologies. The choice of a proper Ontology for the cellular networks' technologies is a very important factor in accomplishing the task of interoperability building and structuring, far beyond the issue of the representation of the inventory of the cellular networks' features.

\subsection{Ontology's Methodology}

Because there is currently no Ontology for the domain of cellular networks' technologies, we are unable to rely on previous work and instead have to develop our own Ontology. We are, however, able to leverage an existing methodology for establishing our Ontology and tailor that methodology to our purpose. The Ontology development process starts by identifying the purpose and scope of the Ontology (step 1). The second step (step 2) is the development of feature analysis for the selected domain (in this case, the domain of cellular networks' technologies). This is followed by (step 3) reasoning and brainstorming about observations and information generated by the feature models to select the commonalities between the three cellular networks' technologies and build a high level Ontology representing these commonalities. The next step (step 4) is to build more detailed Ontologies for each technology. These Ontologies include more essential characteristics at a finer level of granularity. Next (step 5), we used UML [7] to represent the relationships between the three Ontologies. Finally, we documented the Ontology (step 6).

\section{TOWARDS A HOLISTIC DEVELOPMENT APPROACH}

While there has been plenty of research into the development of single aspects in the mobile cellular communication networks field, and some specific frameworks developments, there has been little research into holistic models defining how various cellular networks technologies should interact smoothly. The development of a holistic framework potentially provides clear and explicit understanding of the domain that would generate seamless interoperability between cellular networks' technologies, allowing them to communicate efficiently and reliably with high quality. Additionally, the existence of such framework (figure 2) enhances the discovery of dependencies among different aspects of the cellular networks' technologies. The hope is that it will enable telecommunication researchers to discover various improvements. The long-term goal is to support all aspects of cellular network technologies; however, the immediate goal is to demonstrate the theoretical feasibility of integrating a selected subset of cellular networks' technologies using a holistic framework. 


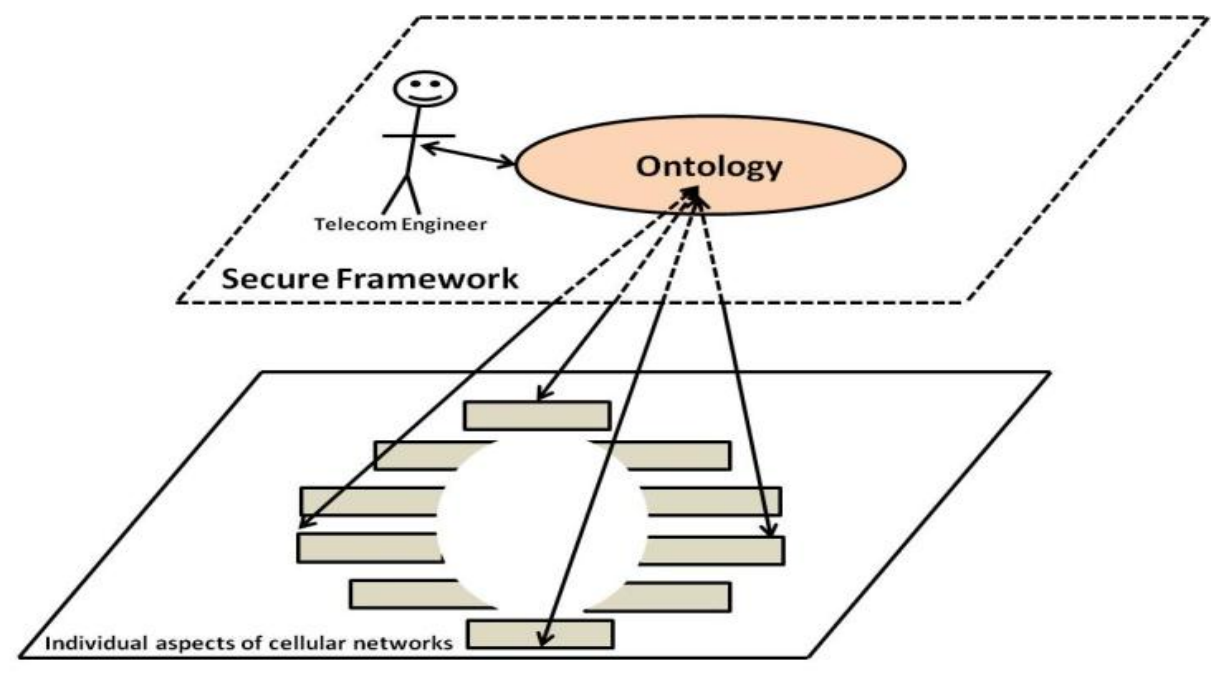

Figure 2: Holistic model for cellular communication networks interaction.

\section{TOWARDS A HOLISTIC DEVELOPMENT APPROACH}

The approach to developing such framework starts by analyzing the structure, inputs, and outputs of a small set of individual cellular technologies, performing a domain analysis (of this subset of technologies) and building a feature model for each of them [4]. All the already mentioned steps will be wrapped up by building an interoperability ontology tailored to cellular networks' technologies [5].

\subsection{Feature Modeling [6]}

To perform a domain analysis of the subset of cellular technologies, we will proceed by producing a feature model for each technology of the domain of interest.

Features are originally used to define software product lines and system families, to identify and manage commonalities and variabilities between products and systems. Attempting to define a feature model for existing cellular networks' technologies allows us to explore, identify, and define the key aspects of existing technologies so that these aspects can be described in Ontology. It is this Ontology that then allows us to improve interoperability between existing cellular networks' technologies.

Our approach for the analysis and the investigation of the structure of inputs, outputs, and relationships of a collection of individual cellular technologies can be characterized as a domain analysis (of this subset of technologies) and the production of feature model of that domain. This technique is well suited for the cellular technologies' features as well as the identification of their essential characteristics. Use of these characteristics in further steps of the research allows them to interoperate.

The feature model is an abstract representation of functionality found in the domain. It is used during domain engineering in order to obtain an abstract view on this functionality, which can be verified against the needs raised by the domain. Therefore, each feature is a relevant characteristic of the domain.
The description of feature models was tied to the introduction of the Feature-Oriented Domain Analysis (FODA) approach in the late eighties. Feature-oriented domain analysis (FODA) is a domain analysis method developed at the Software Engineering Institute (SEI). The method is known for the introduction of feature models and feature modeling. A feature model represents an explicit model of a device or system by summarizing the features and the variation points of the device/system. Feature models include the rationale (a feature should have a note explaining why the feature is included in the model) and the stakeholders for each of feature.

Features are Organized in a hierarchical feature tree with classification (mandatory, optional, alternative, and/or optional alternative features) [8].

$\checkmark$ Mandatory: a mandatory child feature must be included in all the products in which its parent feature is included.

$\checkmark$ Optional: an optional child feature can be optionally included in all products in which its parent feature appears.

$\checkmark$ Alternative: a set of child features are defined as alternative, if only one of them can be selected when its parent feature is part of the product.

$\checkmark$ Or-relation: a set of child features are said to have an Or-relation with their parent when one or more of them can be included in the products in which its parent feature appears.

As an illustration about what already mentioned, figure 3 represents an excerpt of high level features taken from GSM feature diagram. Meanwhile, figure 4 presents a specific portion of the LTE-Advanced feature model. The excerpt presents the LTE-Advanced physical architecture. It presents concepts inherited from both domains: user and infrastructure. Note that each feature is a relevant characteristic of the domain [9]. 


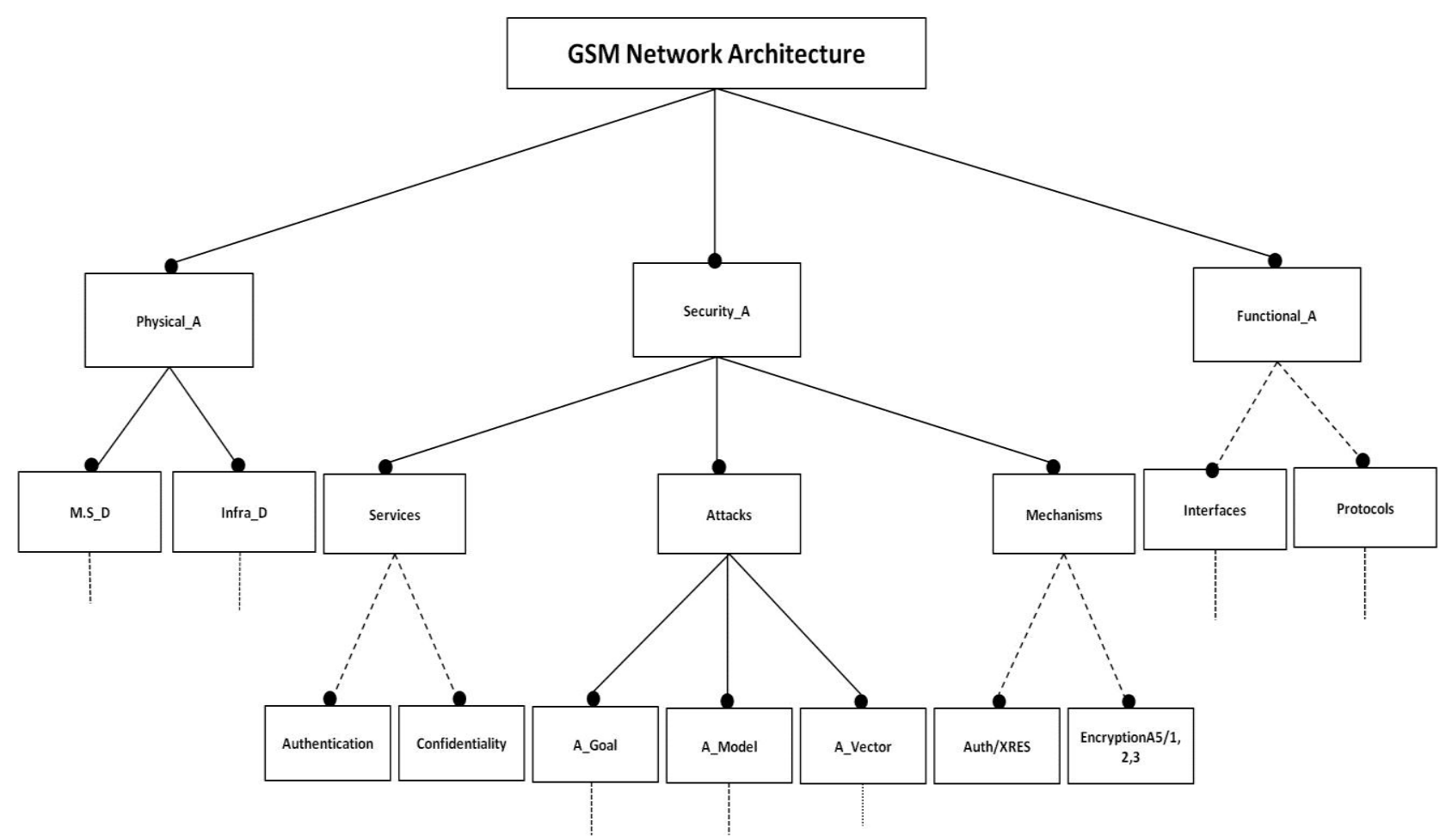

Figure 3. An excerpt of high level GSM feature diagram

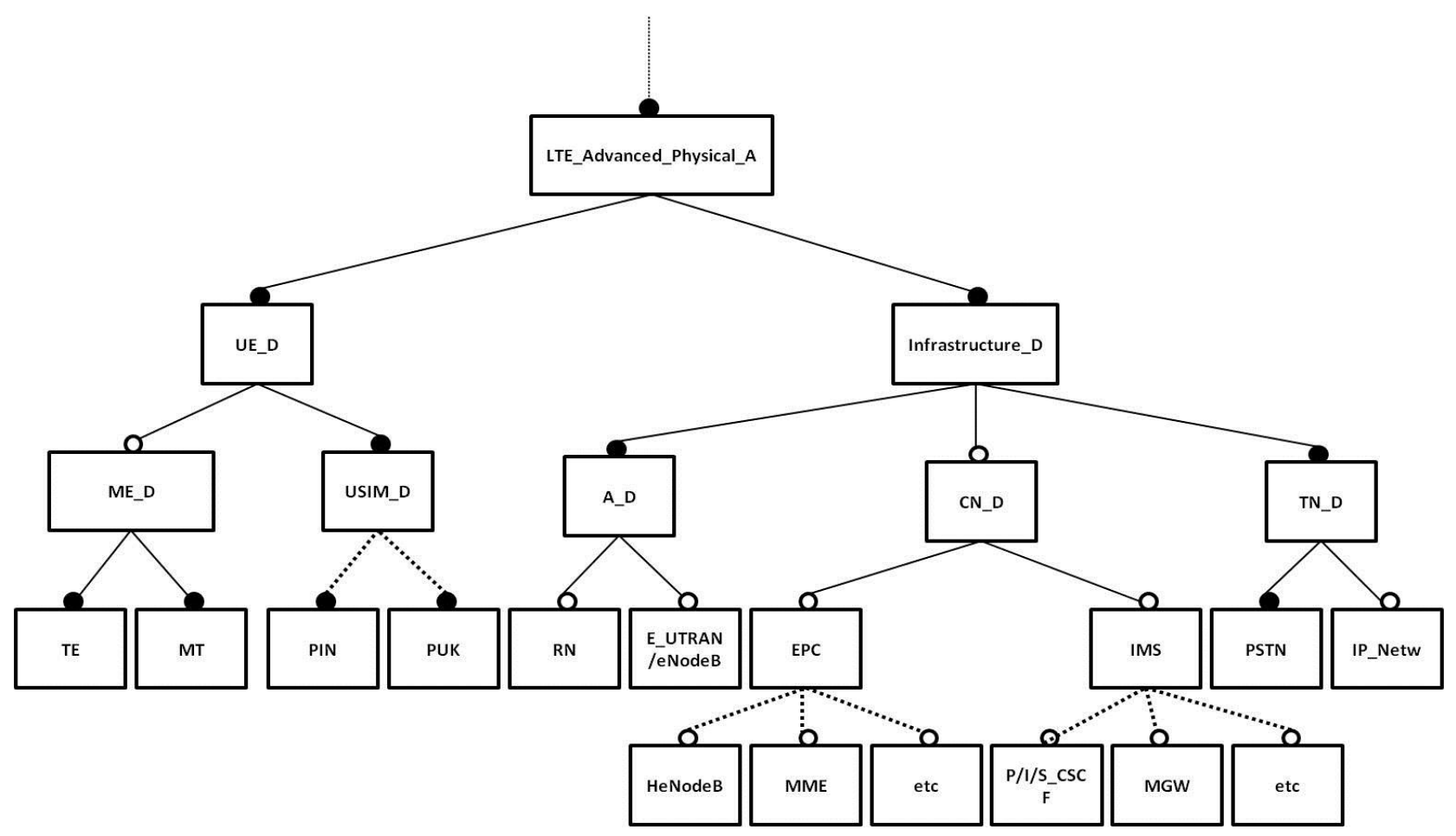

Figure 4. Excerpt of the LTE-Advanced feature model [7]. 
For more clarity, table 1 presents full names of physical architecture features and their descriptions.

\subsection{UML as an Ontology description language}

Like feature model, a well-defined class diagram, part of the Unified Modeling Language (UML), can describe concepts and their relationships in a certain domain of discourse. Both approach have much to offer and can work together most effectively in an integrated environment. It is the reason behind their usage in this ongoing work. The UML is an attractive technique for modeling systems and documenting them [10]. It uses symbols to graphically represent various components and their relationships within a system. UML diagrams are not only made for developers but also for users, and whoever interested in understanding a specific system.
As an important part of UML, class diagrams represent the object oriented view of a system in static way. They are generally used for development purposes [11]. Moreover, they are widely relayed on at the time of system construction. Generally, class diagrams as shown in figure 5, illustrate the existing classes of real world system, and how they are associated to each other in a hierarchical manner.

Each class is represented by a box with three parts: the first one at the top is for the name of the class, the second part is for the attributes of the class (specified by their name, type and visibility) and the third one is for the operations of the class (specified by name, argument list, return type and visibility). For the purposes of annotating our ontologies and to keep figures light and clear, we do list neither the attributes nor the operations. Full diamond is the symbole of Composition (Strongly-owned); meanwhile the empty one represents an Aggregation (non-strongly owned).

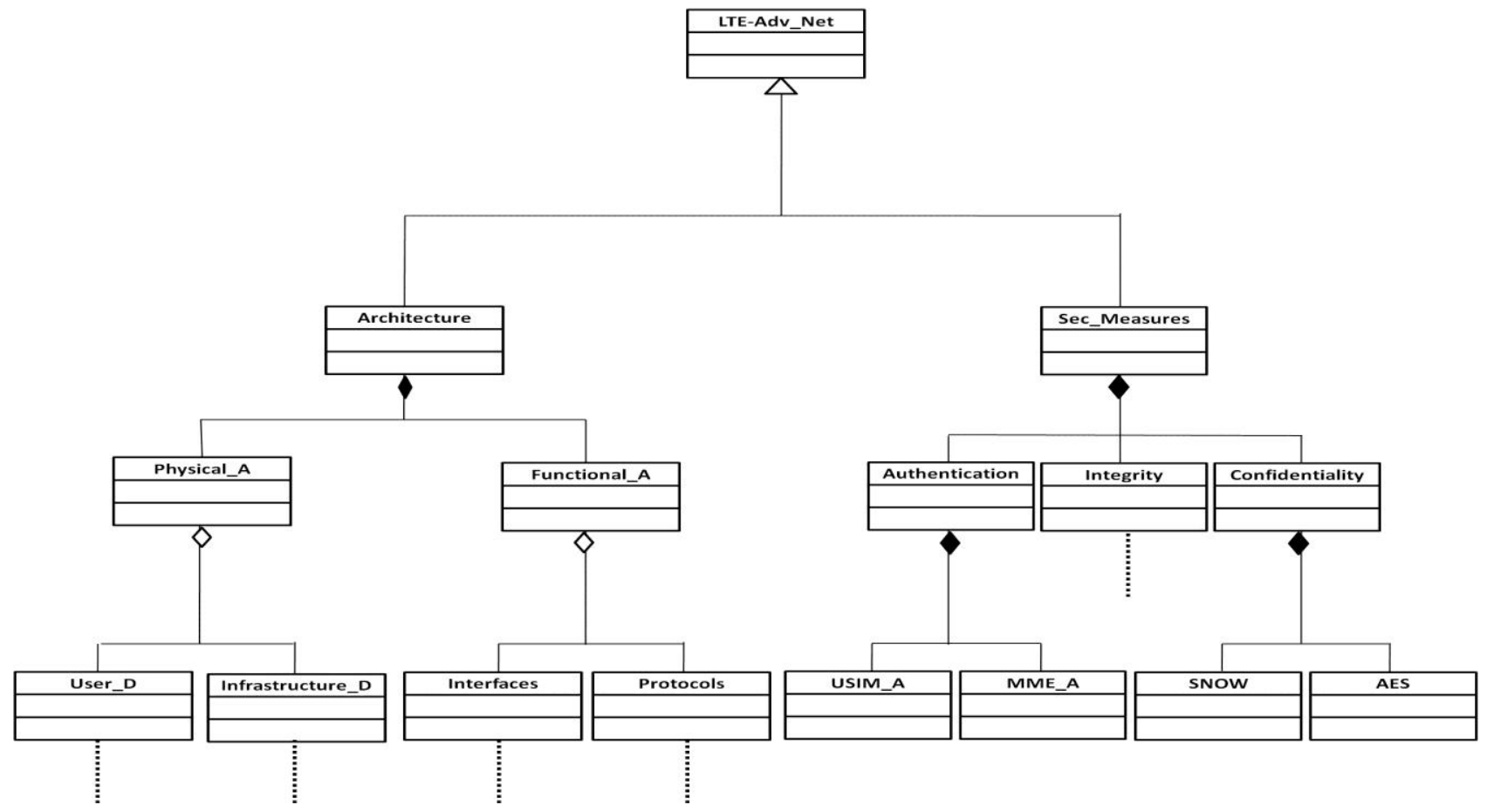

Figure 5. LTE-Advanced network class diagram.

As an important part of UML, class diagrams represent the object oriented view of a system in static way. They are generally used for development purposes [11]. Moreover, they are widely relayed on at the time of system construction. Generally, class diagrams as shown in figure 5, illustrate the existing classes of real world system, and how they are associated to each other in a hierarchical manner.

Each class is represented by a box with three parts: the first one at the top is for the name of the class, the second part is for the attributes of the class (specified by their name, type and visibility) and the third one is for the operations of the class (specified by name, argument list, return type and visibility). For the purposes of annotating our ontologies and to keep figures light and clear, we do list neither the attributes nor the operations. Full diamond is the symbole of Composition
(Strongly-owned); meanwhile the empty one represents an Aggregation (non-strongly owned).

\subsection{Interoperability Ontology}

Different Ontology languages provide different facilities and fulfill the requirements needed for this work. The most recent development in standard Ontology languages is Ontology Web Language (OWL) from the World Wide Web Consortium (W3C) and found in Protégé 4.1. OWL makes it possible to describe concepts [12]. It has a richer set of operators - e.g. intersection, union and negation. It is based on a different logical model which makes it possible for concepts to be defined as well as described. Furthermore, the logical model allows the use of a reasoner which can check whether or not all of the statements and definitions in the Ontology are mutually consistent, and can help to maintain the hierarchy correctly. 
The already mentioned reasons certify the high-quality of the tool. Protégé 4.1, OWL was chosen in order to make the descriptions of concepts, attributes, and instances formal, so as the knowledge can be machine-readable and reasoningautomated. Figure 6, is a screen capture of the three Ontologies' classes generated by protégé 4.1 OWL environment.

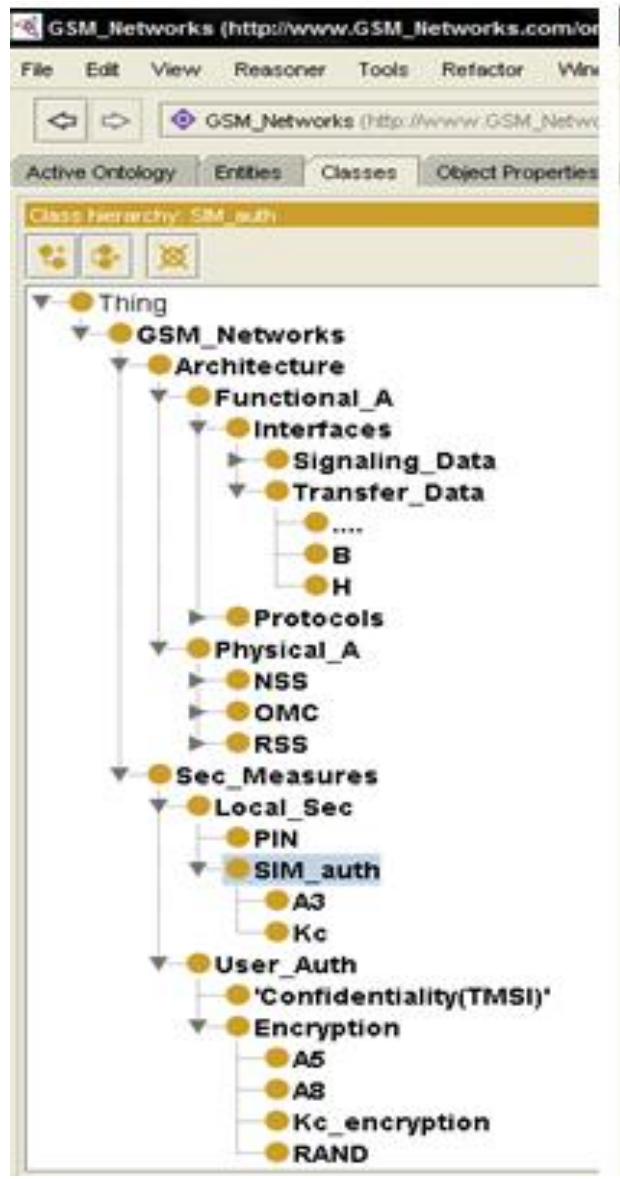

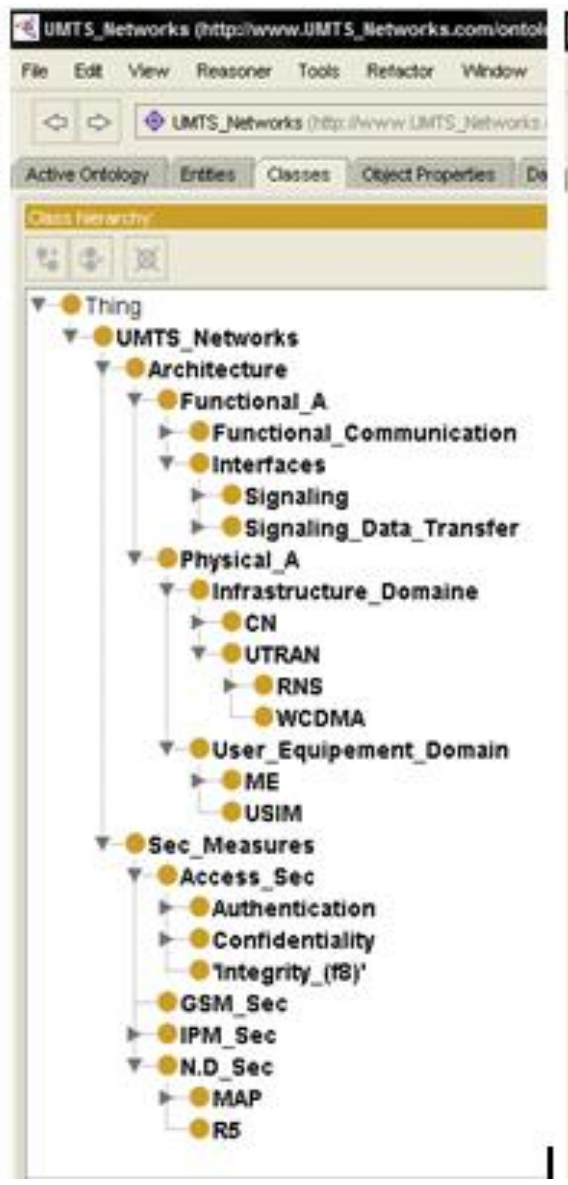

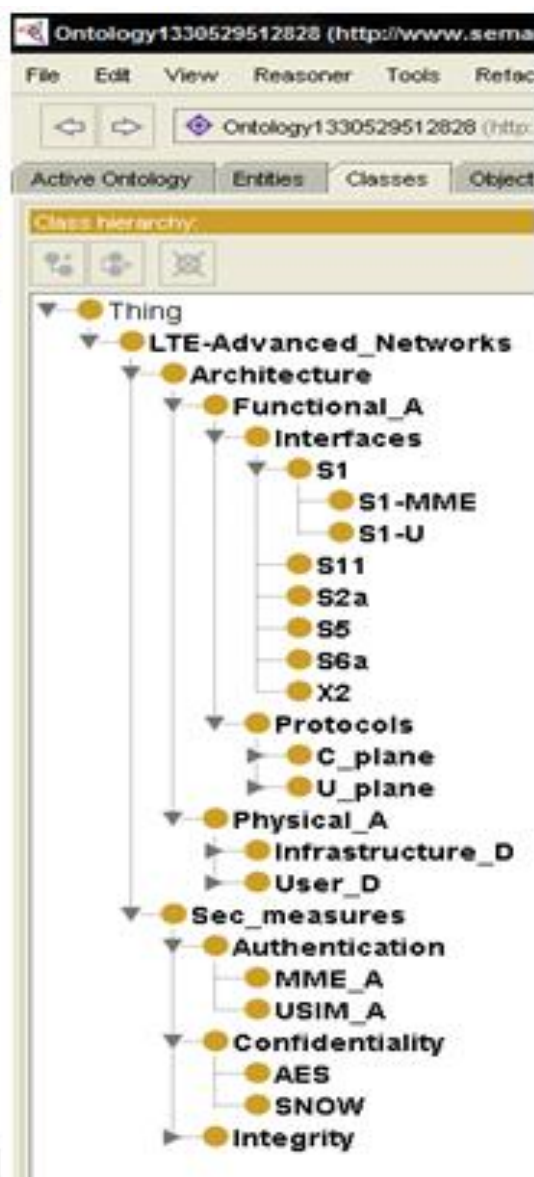

Figure 6. Screen capture of the three ntologies' classes.

The relationships between all three Ontologies are identified and annotated. The reason for this is to formulate inter-relationships between them. Figure 7 illustrates the general UML structure that was used to annotate how all three Ontologies were related.

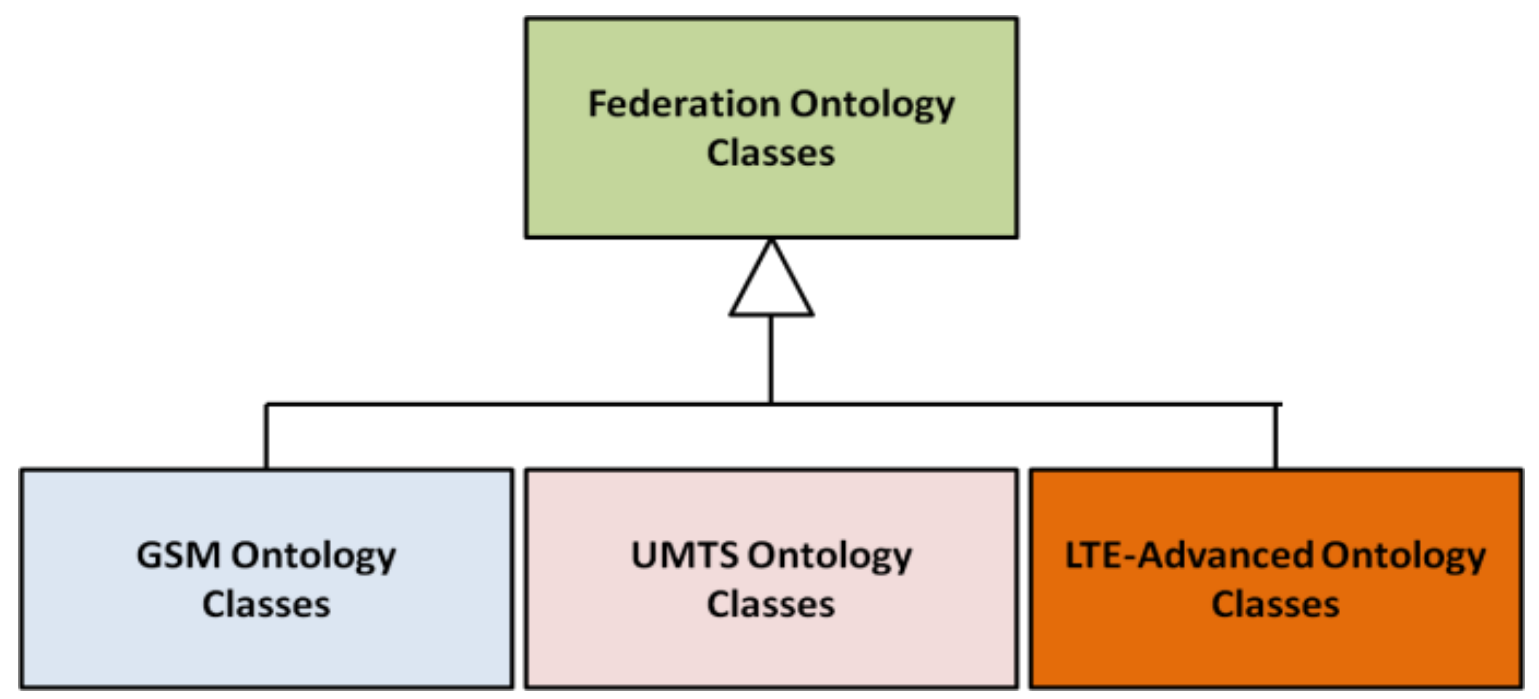

Figure 7. UML Ontologies' inter-relationship. 
Table.1 LTE-Advanced physical architecture feature list [2].

\begin{tabular}{|c|c|c|}
\hline Feature & Full name of the feature & Description \\
\hline Physical_A & Physical Architecture & \\
\hline User_D & User Domain & \\
\hline RN & Relay Node & Relays are designed for coverage extension \\
\hline MT & Mobile Termination & $\begin{array}{l}\text { Mobile termination in the network of "B" is referred to when } \\
\text { calls are routed to operator "B" via the network of operator } \\
\text { "A", in order to be delivered to the end customer in the } \\
\text { mobile network of "B". }\end{array}$ \\
\hline Infrastructure_D & Infrastructure Domain & \\
\hline E_UTRAN & $\begin{array}{l}\text { Evolved Universal Terrestrial } \\
\text { Radio Access Network }\end{array}$ & $\begin{array}{l}\text { EUTRAN implements the LTE-Advanced access network as } \\
\text { a network of eNBs. }\end{array}$ \\
\hline HeNodeB & Home enhanced Node B & An evolved network component that serves one femtocell \\
\hline eNodeB & enhanced Node B & $\begin{array}{l}\text { An evolved network component that serves one cell. } \\
\text { The eNB is responsible for many functions including: } \\
\text { Radio Resource Management, IP header compression and } \\
\text { user data encryption, the scheduling and allocation of uplink } \\
\text { and downlink radio resources, and coordinating handover } \\
\text { with neighboring eNBs. eNBs can communicate with } \\
\text { multiple gateways for load sharing and redundancy. }\end{array}$ \\
\hline EPC & Evolved Packet Core & $\begin{array}{l}\text { LTE-Advanced's packet domain is a flat all-IP system } \\
\text { designed for: much higher packet data rates, significantly } \\
\text { lower-latency, the ability to optimize packet flows within all } \\
\text { kinds of operational scenarios having to do with bandwidth } \\
\text { rationing and charging schemes, explicit support for multiple } \\
\text { radio access technologies in the interests of seamless } \\
\text { mobility, and greater system capacity and performance. }\end{array}$ \\
\hline PDNGW & $\begin{array}{l}\text { Packet Data Network } \\
\text { Gateway }\end{array}$ & $\begin{array}{l}\text { Acts as a default router for the UE, and is responsible for } \\
\text { anchoring the user plane for mobility between some } 3 \mathrm{GPP} \\
\text { access systems and all non-3GPP access systems. }\end{array}$ \\
\hline MME & Mobility Management Entity & $\begin{array}{l}\text { Supports many functions for managing mobiles and their } \\
\text { sessions. }\end{array}$ \\
\hline HeNBGW & H(e)NB Gateway & $\begin{array}{l}\text { Is a mobile operator's equipment (usually physically located } \\
\text { on mobile operator premises) through which the H(e)NB gets } \\
\text { access to mobile operator's core network. } \\
\text { HeNBGW serves as a concentrator for the C-Plane, } \\
\text { specifically the S1-MME interface. The HeNBGW may } \\
\text { optionally terminate the user plane towards the HeNB and } \\
\text { towards the SGW, and provide a relay function for relaying } \\
\text { User Plane data between the HeNB and the S-GW. The } \\
\text { HeNB GW supports NAS Node Selection Function (NNSF). }\end{array}$ \\
\hline SGW & The Serving Gateway & $\begin{array}{l}\text { Is responsible for anchoring the user plane for inter-eNB } \\
\text { handover and inter-3GPP mobility. }\end{array}$ \\
\hline HSS & Home Subscriber Server & $\begin{array}{l}\text { Is the master database that stores subscription-related } \\
\text { information to support call control and session management } \\
\text { entities. }\end{array}$ \\
\hline IMS & IP Multimedia Subsystem & It includes charging, billing and bandwidth management. \\
\hline MGCF & $\begin{array}{l}\text { Media Gateway Control } \\
\text { Fonction }\end{array}$ & $\begin{array}{l}\text { Communicates with the Call Session Control Function } \\
\text { (CSCF) and controls the connections for media channels in } \\
\text { an IMS-MGW. It performs protocol conversion between } \\
\text { ISDN User Part (ISUP) and the IMS call-control protocols. }\end{array}$ \\
\hline P/I/S_CSCF & proxy/interrogating/serving & Is the first contact point in IMS and interacts with GGSN \\
\hline
\end{tabular}




\begin{tabular}{|l|l|l|}
\hline & call session control function & (Gateway GPRS Support Node). \\
\hline MGW & Media Gateway & $\begin{array}{l}\text { It's a tool or service that transforms media flow between } \\
\text { network telecommunication. }\end{array}$ \\
\hline
\end{tabular}

\section{CONTRIBUTIONS}

The telecommunication contributions represented in this study are:

1. An initial investigation and analysis of the structure, and architecture of three cellular networks' technologies, and the identification of essential characteristics of each of them.

2. The completion of a domain analysis of these technologies and the production of a feature model for everyone.

3. The preparation for an identification of the commonalities (from the point of view of architectural characteristics) between these networks' technologies.

4. The use of feature models as a key asset to manage the commonalities and the variabilities of the cellular networks' technologies. The common features then form the basis for the interoperability Ontology of the domain of interest.

5. The description of cellular networks' structure using a top-down approach. The networks were logically divided into domains and strata.

6. The use of UML to describe concepts and relationships in the cellular communication domain.

\section{CONCLUSION}

Improvements to individual parts of the cellular communication networks without regard to the interrelationship between them do not guarantee overall improvement in interoperability. The Secure Framework for mobile cellular networks (SFMCN) will be holistic in nature. It will be able to track networks' artifacts and relationships throughout the entirety of the cellular communication networks innovations. While there is no "silver bullet" that will solve all cellular networks interoperability challenges and problems, the SFMCN will provide an improved model upon which to develop safe, reliable and interoperable environment for mobile cellular communication. Moreover, SFMCN demonstrates that establishing a theoretical framework allowing existing heterogeneous cellular communication networks to understand each other is technically feasible.

\section{REFERENCES}

[1] Hasni NEJI, Ontology for Cellular Communication, Proceedings of Tunisian Japanese Symposium on Science, Society and Technology, Tunisia, November $11-13,2011$

[2] N. Hasni, R. Bouallegue, "Roadmap for Establishing Interoperability of Heterogeneous Cellular Network Technologies -1-", International Journal of Computer Science and Network Security, VOL.12 No.4, pp. $92-$ 103, April 2012.

[3] N. Hasni, R. Bouallegue, "Roadmap for Establishing Interoperability of Heterogeneous Cellular Network Technologies -2-", Journal of Signal and Information Processing, VOL.3 No.3, August 2012.

[4] Czarnecki, K. and Eisenecker, U., Generative Programming Methods,Tools, and Applications, Addison- Wesley, 2000.

[5] Uschold, M. and Gruninger, M., "Ontologies: Principles, Methods and Applications," Knowledge Engineering Review, Vol. 11, No. 2, June 1996.

[6] Hasni, N., "Towards an interoperability ontology for software development tools" Master's Thesis, Computer Science Department, Naval Postgraduate School, Monterey, CA, March 2003.

[7] Cranefield, S. and Purvis, M., "UML as an Ontology Modelling Language", Proceedings of the IJCAI'99 Workshop on Intelligent Information Integration, Sweden, 1999.

[8] Geyer, L., "Feature Modeling Using Design Spaces," Proceedings of $1^{\text {st }}$ German Workshop on Product Line Software Engineering, Kaiserslautern, Germany, November 2000 .

[9] 3rd Generation Partnership Project; Technical Specification Group Services and System aspects; Technical Specification Group working methods (Release 10), 03/2011.

[10] OMG Unified Modeling LanguageTM (OMG UML), Superstructure Version 2.2 February 2009.

[11] Kogut, P., Cranefield, S., Hart, L., Dutra, M., Baclawski, K., Kokar, M., and Smith, J., "UML for Ontology development," Knowledge Engineering Review, Vol. 17, Issue 1, March 2002, pp. 61-64.

[12] Matthew Horridge and al, A Practical Guide To Building OWL Ontologies Using Prot'eg'e 4 and COODE Tools, Edition 1.2, The University Of Manchester, March 13, 2009 